\title{
Astronomía práctica en la Enseñanza Fundamental
}

\author{
Astronomy practice in Elementary School \\ Prática de Astronomia no Ensino Fundamental
}

Recebido: 28/07/2021 | Revisado: 02/08/2021 | Aceito: 05/08/2021 | Publicado: 10/08/2021

Jean Carlos Soares de Oliveira

ORCID: https://orcid.org/0000-0002-2890-0858 Instituto Federal de Educação, Ciência e Tecnologia de Mato Grosso, Brasil E-mail: soaresjeancarlos@gmail.com

Edward Bertholine de Castro

ORCID: https://orcid.org/0000-0003-2316-4190 Universidade Federal de Mato Grosso, Brasil E-mail: vava67@gmail.com

Eduardo Ribeiro Mueller

ORCID: https://orcid.org/0000-0002-6486-919X Universidade Federal de Mato Grosso, Brasil E-mail: edurmueller@hotmail.com Geison Jader Mello

ORCID: https://orcid.org/0000-0002-0991-2327 Instituto Federal de Educação, Ciência e Tecnologia de Mato Grosso, Brasil E-mail: geison.mello@cba.ifmt.edu.br

\begin{abstract}
Resumen
Este trabajo presenta la intervención realizada en la Escuela Estadual Francisco Araújo Barreto, en el Municipio de Jaciara - MT - Brasil, e tu meta fue enseñar astronomía con clases teóricas y prácticas, con la aplicación del contenido en la disciplina de Ciencias con los alumnos del $6^{\circ}$ y $9^{\circ}$ año de la Enseñanza Fundamental. El tema aborda el contenido de Astronomía de forma teórica y práctica, a fin de llevar a un aprendizaje que sea significativo. Durante 5 lecciones los temas de las nociones básicas de Astronomía fueron abordados con clases expositivas y prácticas. Las estrategias que apuntan a facilitar la enseñanza-aprendizaje fueron las siguientes: al inicio de las dos primeras clases se aplicó un cuestionario a fin de levantar datos sobre el conocimiento previo de los alumnos, las clases prácticas se aplicaron de forma que todos los alumnos producían su material para el crecimiento y enriquecimiento de conocimiento, por fin los alumnos fueron llevados al estadio municipal para conocer un planetario, de forma expositiva y teórica la clase tuvo una importancia de gran relevancia para los alumnos, que al final de las 5 clases respondieron a un cuestionario alcanzando el objetivo esperado. Los resultados alcanzados permitieron inferir que aulas de clases teóricas y prácticas de astronomía fueron positivas, motivando a los estudiantes a participar en las clases y actividades, y además, posibilitando un aprendizaje significativo.
\end{abstract}

Palabras clave: Enseñanza Fundamental; Astronomía; Enseñando.

\begin{abstract}
This work presents the intervention performed in the State School Francisco Araujo Barreto, in the municipality of Jaciara - MT, with the application of astronomy content in Science discipline with the students of 6th and 9th grade of elementary school. The theme addresses the astronomy content theoretically and practices in order to lead to learning that is meaningful. For 5 classes the topics of basics of Astronomy were addressed with lectures and practices. Strategies to facilitate the teaching-learning were as follows at the beginning of the first two classes were applied a questionnaire to collect data on students' prior knowledge, the practical classes were applied so that all students produce their material to the growth and enrichment of knowledge, finally the students were taken to the municipal stadium to meet a planetarium, expository and theoretically the class had an importance of great importance to students, at the end of the 5 classes completed a questionnaire reaching the expected goal. The achieved result showed that theoretical as aulas and de astronomy practices were positive, motivating students to participate in classes and activities, and also enabling meaningful learning.
\end{abstract}

Keywords: Elementary school; Astronomy; Teaching.

\section{Resumo}

Este trabalho apresenta a intervenção realizada na Escola Estadual Francisco Araújo Barreto, no Município de Jaciara - MT - Brasil, e seu objetivo foi o ensino de Astronomia com aulas teóricas e práticas, com a aplicação dos conteúdos na disciplina de Ciências com os alunos do $6^{\circ}$ e $9^{\circ}$ ano do Ensino Básico. O tema aborda o conteúdo da Astronomia de forma teórica e prática, de maneira a conduzir a uma aprendizagem significativa. Durante 5 aulas foram abordados os tópicos das noções básicas de Astronomia com aulas teóricas e aulas práticas. As estratégias destinadas a facilitar o 
ensino-aprendizagem foram as seguintes: no início das duas primeiras aulas foi aplicado um questionário para recolher dados sobre os conhecimentos prévios dos alunos, as aulas práticas foram aplicadas de forma a que todos os alunos produzissem os seus material para o crescimento e enriquecimento do conhecimento, finalmente os alunos foram levados ao estádio municipal para ver um planetário, de forma expositiva e teórica a aula teve uma importância de grande relevância para os alunos, que ao final das 5 aulas responderam a um questionário atingindo o objetivo esperado. Os resultados alcançados permitiram inferir que as aulas teórico-práticas de astronomia foram positivas, motivando os alunos a participarem das aulas e atividades, além de possibilitar uma aprendizagem significativa.

Palavras-chave: Ensino Fundamental; Astronomia; Ensino.

\section{Introducción}

La enseñanza de la Astronomía a veces ha sido aplicada superficialmente en las instituciones de enseñanza en Brasil actualmente, tenemos como ejemplo nuestro propio curso de Licenciatura en Ciencias de la Naturaleza, donde no tiene la Astronomía como disciplina curricular del curso. Con base en el libro Ciencias Naturales, $6^{\circ}$ año, $3^{a} \mathrm{ed}$. (Santana \& Figueiredo Neto, 2009), el material didáctico es para alumnos del $6^{\circ}$ año, donde es presentado como contenido programático" TERRA: Forma y Ubicación en el Espacio ", y "Algunas Características de nuestro Planeta", contenido presentado respectivamente en el inicio del libro, tiene como énfasis dimensiones de los astros celestes, movimientos de la tierra, día y noche y etc, conceptos básicos de la astronomía que los profesores están teniendo dificultades para abordar en el aula, porque el contenido no les fue presentado de forma significativa en su graduación, eso hace que el tema sea aplicado de forma superficial en la enseñanza fundamental, resultando en poco aprovechamiento y absorción por parte de los alumnos.

El resultado que podemos ver en la publicación del PISA 2000 - (Programa Internacional para Evaluación de Estudiantes), en que Brasil quedó el $43^{\circ}$ lugar entre los países participantes. Ante la falta de dominio del tema, observamos que poco se habla en el ámbito nacional, y la Astronomía tratada en las escuelas no pasa de lecturas del material didáctico realizadas en aulas. Tenemos hoy algunas instituciones en Brasil que ofrecen cursos para formación continuada de profesores en el área, pero pocos profesores se interesan en ir atrás para perfeccionar el proceso el conocimiento, ante lo que puede ser hecho para la mejora de este cuadro, es hacer las clases participativas, clases que involucran al alumno, clases prácticas y clases expositivas pueden colaborar significativamente para la expansión del contenido, haciendo la invitación para el alumno que consecuentemente irá tener una participación más amplia con una mejor absorción del contenido (Damasceno Júnior \& Romeu, 2018).

En la actualidad, los conceptos básicos de la astronomía se están presentando superficialmente para alumnos de enseñanza fundamental, sin duda el contenido que esta en la matriz curricular de la disciplina de Ciencias Naturales, no está siendo aplicado de forma que sea absorbido significativamente por el alumno para una buena interpretación, con lo que el tema se vuelve irrelevante en la continuidad escolar y en el futuro académico, tal situación no ocurre sólo en la formación escolar, pero también tiene gran reflejo en la formación académica, el hecho de que la disciplina no forma parte del currículo de los cursos para la formación de profesores de ciencias naturales implica en un proceso bajo entendimiento e interpretación del profesor que consecuentemente no pasa por delante porque el mismo no tiene el conocimiento necesario.

La disciplina "Ciencias Naturales" es presentada para el alumno en el $6^{\circ}$ año de la enseñanza fundamental, momento propicio para la fijación del saber, por estar en fase de desarrollo, la absorción de conocimiento es aún mayor, pero desafortunadamente muchas veces la disciplina no es le ha presentado de forma que llame su atención, a fin de despertar el interés por el conocimiento.

De esta forma, el objetivo de este trabajo fue enseñar astronomía con clases teóricas y prácticas, a fin de despertar la curiosidad del alumno para investigación del espacio, de forma que no queden sólo en lecturas de literaturas dentro del aula, sino practicar para realmente aprender a interpretar el material didáctico propuesto, utilizando el material didáctico ofrecido por la Olimpiada Brasileña de Astronomía - OBA, y tendrá como destaque clases expositivas y prácticas. 
Los objetivos específicos son: presentar actividad que representa las distancias y el tamaño de los planetas proporcional a los tamaños propuestos; el movimiento de los planetas; mostrar la importancia que el estudio del ESPACIO significa, para la evolución de nuevas tecnologías que beneficie a la humanidad; despertar la curiosidad de las investigaciones de los astros celestes.

\section{La Enseñanza de las Ciencias de la Naturaleza}

Con el fin de colaborar para la comprensión del mundo, las Ciencias Naturales se aplican en los nueve años de la Enseñanza Fundamental brasileño, presentando conceptos y procedimientos en las áreas de Química, Física y Biología, que se distribuyen en la forma de construcción de saber, los temas se distribuyen según la complejidad pedagógica del contenido (Brasil, 2017).

Cada profesional de educación tiene su individualidad a la hora de aplicar el contenido, por eso vemos constantes cambios en la aplicación, y la búsqueda de la mejor forma de llevar al alumno a absorber el aprendizaje propuesto.

Para Nóvoa (2009):

La educación vive un tiempo de grandes incertidumbres y de muchas perplejidades. Sentimos la necesidad del cambio, pero no siempre hemos podido definir el rumbo. Hay un exceso de discursos, redundantes y repetitivos, que se traduce en una pobreza de prácticas (Nóvoa, 2009, p.27).

En la enseñanza de las ciencias se hace necesario la aplicación de clases práctica, pues una de las mayores dificultades que podemos observar por parte de los alumnos es en la interpretación del contenido, y en conseguir conciliar con lo cotidiano del día a día aquel contenido visto por ellos de forma compleja en el aula, podemos observar en la unión de la clase teórica con la clase práctica un mejor rendimiento y absorción de conocimiento, pues en la teoría los asuntos son tratados de forma más científica y que muchas veces estamos citando cosas rutinarias sin utilizar un vocabulario más complejo y, distante de la realidad de los alumnos (Nóvoa, 2009).

\subsection{Aprendizaje Significativo}

El aprendizaje significativo ocurre cuando la disponibilidad del alumno para la construcción del saber, y establecer un vínculo entre lo que ya sabe y en qué está aprendiendo, con la utilización de instrumentos que ya conoce a fin de obtener mayor comprensión (Moreira, 2006). Según la Base Curricular Nacional Común (Brasil, 2017),

é imprescindível que eles (os alunos) sejam progressivamente estimulados e apoiados no planejamento e na realização cooperativa de atividades investigativas, bem como no compartilhamento dos resultados dessas investigações. Dessa forma, o processo investigativo deve ser entendido como elemento central na formação dos estudantes, em um sentido mais amplo, e cujo desenvolvimento deve ser atrelado a situações didáticas planejadas ao longo de toda a educação básica, de modo a possibilitar aos alunos revisitar de forma reflexiva seus conhecimentos e sua compreensão acerca do mundo em que vivem (BRASIL, 2017, p. 322 - grifo nosso).

En la enseñanza de las ciencias se hace necesario la aplicación de clases práctica, pues una de las mayores dificultades que podemos observar por parte de los alumnos es en la interpretación del contenido, y en conseguir conciliar con lo cotidiano del día a día aquel contenido visto por ellos de forma compleja en el aula, podemos observar en la unión de la clase teórica con la clase práctica un mejor rendimiento y absorción de conocimiento, pues en la teoría los asuntos son tratados de forma más científica y que muchas veces estamos citando cosas rutinarias sin utilizar un vocabulario más complejo y, distante de la realidad de los alumnos (Nóvoa, 2009). 
El aprendizaje significativo establece un equilibrio entre la teoría y la práctica, enfatizada en las actividades rutinarias en que se aplica automáticamente las actividades pedagógicas que cada disciplina exige para la comprensión del contenido, de forma que el alumno memorice en la forma práctica y absorción mayor parte del contenido. Pero sólo presentar el contenido atractivo no es suficiente si no hay empeño en aprender por parte del alumno, pues el conocimiento no es adquirido sólo en el aula, sino con investigaciones y lecturas periódicas para que haya cuestionamientos y consecuentemente la enseñanzaaprendizaje (Ausubel, 1982).

Casi cualquier profesor alguna vez durante el período de docencia probablemente ya oyó de algún alumno la siguiente pregunta: "¿En qué momento de mi vida voy a usar eso?". El aprendizaje significativo trabaja dentro del conocimiento previo del alumno, para aplicar el contenido dentro de alguna situación rutinaria vivida por el alumno, con el objetivo de la ficción del saber por parte del alumno. Para Ausubel (1982):

El aprendizaje es mucho más significativo a medida que el nuevo contenido se incorpora a las estructuras de conocimiento de un alumno y adquiere significado para él a partir de la relación con su conocimiento previo. (Ausubel, 1982, p.38).

\subsection{Astronomía en la Educación Básica}

En el transcurso de los años la disciplina de Astronomía viene disminuyendo la carga horaria y en algunos cursos de ciencias ya no forma parte de la cuadrícula curricular, y la formación acaba quedando incompleta, con lo que la percepción de interpretación del contenido disminuye significativamente haciendo que el tema no se aborda, y / o se enseña de la forma necesaria (Langhi \& Nardi, 2009).

El motivo por el cual la astronomía está a lo largo del tiempo perdiendo espacio como disciplina curricular es recurrente las deficiencias en las metodologías de enseñanza del contenido. Otra deficiencia que afecta el abordaje del tema "Astronomía", se da por el hecho de profesores que tienen la formación en Ciencias Biológicas, que asumen grupos del 6 al 9 , y la disciplina de Astronomía no forma parte de la rejilla curricular en la graduación del As (Tignanelli, 1998).

Se observa que poco es absorbido y poco practicado en el aula, y eso acarrea en una clase con pocas dinámicas y prácticas y que no despiertan la curiosidad de los alumnos para el tema, haciéndolo de poca relevancia para su formación, según datos del Programme for (PISA), que evalúa cada dos años alumnos de enseñanza básica, con la participación de 43 países, un total de 228.784 alumnos, entre ellos alumnos brasileños de enseñanza básica. La evaluación que abortó el tema en el año 2000, apuntó que los alumnos brasileños tuvieron un promedio de acierto menor que los demás participantes, teniendo en cuenta que la evaluación fue aplicada hace 15 años, y que en los últimos años algunos incentivos fueron creados en el área de la educación, esperamos mejores resultados en 2016, donde el PISA y en el caso de que se trate de una "astronomía" en su evaluación (Barroso \& Borgo, 2011).

Con base en grandes investigaciones espaciales podemos gozar de diversos recursos que facilita nuestro cotidiano, respuestas para muchos cuestionamientos referentes a la existencia humana.

Para Gaio (2009) "Aunque a veces no podemos comprender de inmediato qué importancia puede tener lo que aprendemos sobre el Universo, somos una especie en busca de conocimiento" (Gaio, 2009, p.103)

La búsqueda por el saber es constatada y todo conocimiento es construido en formatos de escalones de una escalera, donde cada conocimiento crea una base para el próximo conocimiento que vamos a absorber, pues somos racionales y empezamos a realizar nuestras investigaciones individuales desde nuestro nacimiento, el nuevo sucede cada día (Freire, 1996).

En cuanto a lo aprendido, queremos aprender, hasta ahora no se ha encontrado el inicio y tampoco se ha encontrado el fin del universo, sólo sabemos que hay miles de millones de galaxias y muchos otros miles de millones de galaxias para ser 
descubiertas y estudiadas, o sea por la ciencia, porque para el diccionario Aurelio, Ciencias significa, "Conjunto metódico de conocimientos obtenidos mediante la observación y la experiencia" (Mourão, 2000; Mourão; 2003).

\section{Material e Metodos}

\subsection{Descripción de la Escuela}

La Escuela Estadual Francisco Araújo Barreto, sito a la calle Acues, 1148, Barrio Planalto, en el municipio de JaciaraMT, la institución de enseñanza es mantenida por la Red Oficial de Enseñanza del Estado de Mato Grosso, a través de la Secretaría de Estado de Educación; fue creada por el Acta n ${ }^{\circ}$ 014/83 publicada en el Diario Oficial de 06 de diciembre de 1983, página 23 y por el Decreto n ${ }^{\circ} 843$ de 02 de abril de 1996, publicado en Diario Oficial n ${ }^{\circ} 21.873$ de 02 de abril de 1996 con la denominación de la Escuela Estadual de $1^{\circ}$ Grado "Francisco Araújo Barreto" y recibió la actual denominación a través del Decreto ${ }^{\circ} 1.826$ de 11 de octubre de 2000, Diario Oficial de 11 de octubre de 2000, página 01; que se autoriza a funcionar la Enseñanza Fundamental Regular por la Resolución 049/00-CEE / MT de 29 de febrero de 2000, publicada en el Diario Oficial de 20/03/2000, página 21 y reconocida por la Ordenanza 2572/91, publicada en el Diario Oficial de 04 de julio de 1991. Autorizada a funcionar la Enseñanza Fundamental Modalidad Educación de Jóvenes y Adultos por la Resolución 107/01-CEE / MT, publicada en el Diario Oficial de 17 de abril de 2001, página 05 (Figura 1).

Figura 1. Fachada de la Escuela Estatal Francisco Araújo Barreto, donde se aplicó la intervención.

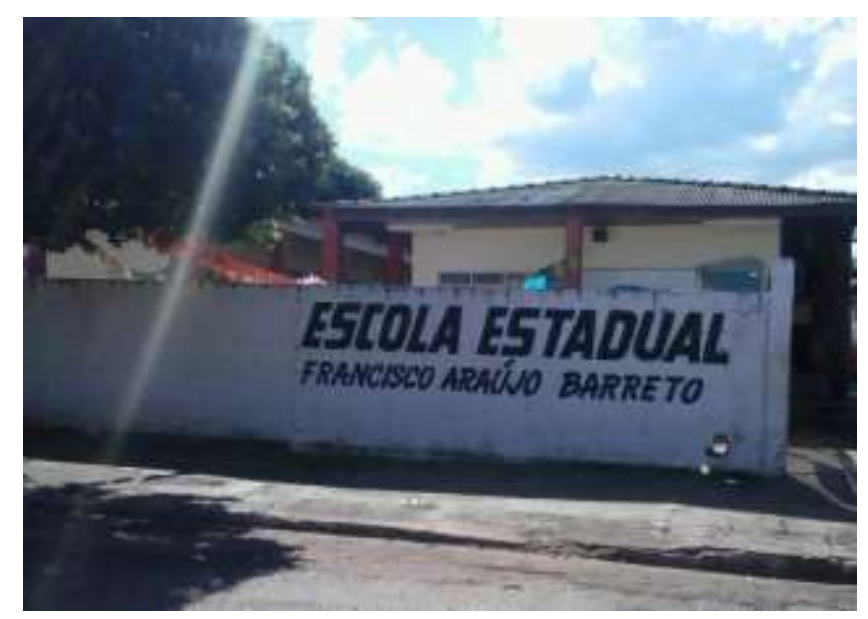

Fonte: Autores.

En el marco de las Leyes Educativas, la institución funciona como Enseñanza Fundamental de 09 años, en la modalidad Ciclos de Formación Humana, con atención a la comunidad en dos períodos: Matutino $\left(6^{\circ}\right.$ al $9^{\circ}$ año $)$ y Vespertino ( $1^{\circ}$ al $5^{\circ}$ año), la escuela también participa del Programa Más Educación, donde los alumnos en el horario alternado participan de varios talleres como: futsal, pintura, bordado, danza, capoeira, entre otras.

\subsection{Metodologia de Pesquisa}

Las intervenciones formativas de carácter teórico-práctico, como en este trabajo, juegan un rol significativo en la toma de decisiones del alumno respecto a su aprendizaje, favoreciendo mucho el avance de la curiosidad ingenua a la curiosidad epistemológica (Freire, 1996).

La acción de investigar asociada a la acción de enseñar, acción-investigación, es un tipo de metodología cualitativa en educación en la que el investigador se involucra activamente en la causa inherente a su investigación (Bogdan \& Biklen, 1994). De esta metodología de investigación se origina un constante entrelazamiento de acción y reflexión sobre la acción, 
evidenciado principalmente en el diálogo del docente con el alumno sobre el conocimiento, garantizado desde la elección del alumno por dialogar. Esta lección, por parte del alumno, tiene un sesgo complejo, y su éxito está subordinado a la elección metodológica del profesor a la hora de recurrir a la práctica. En el ámbito de la práctica, ya sea dentro o fuera del aula de clases, el nivel de reflexiones, preguntas y calidad de la intervención influyen en en el proceso de aprendizaje, cada vez más significativo.

Orientada a lograr resultados que promuevan la superación de las condiciones sociales de los sujetos involucrados en la investigación, y aquí vemos a la escuela desempeñando este papel, la investigación acción es una metodología basada en el proceso de colaboración comunicativa en el que se toman en serio todos los aportes. En él, el significado construido en el proceso de investigación conduce a la construcción de nuevos significados, y estos generan una acción social (Chizzotti, 2006). Leer e interpretar un fenómeno natural, como propone la Astronomía en el estudio de las estrellas, puede no representar, en sí mismo, el sesgo social mencionado por el autor. Aquí tenemos que verla, la Astronomía, no sólo como contenido, sino como conocimiento escolar; este conocimiento, como todos los demás del contexto escolar, se deriva de una construcción de significados de extrema repercusión social.

Además, hay autores que corroboran nuestra elección del abordaje cualitativo para esta investigación. Pereira y col. (2018) nos dice que los métodos cualitativos buscan valorar la interpretación del investigador con sus opiniones sobre el fenómeno en estudio. Flick (2013) defiende que la investigación cualitativa no se moldea en la medida, como en muchos casos de las ciencias naturales, y como en este caso; cuando lo adoptamos, no nos interesa estandarizar una situación, tampoco garantizar la representatividad mediante un muestreo aleatorio de los participantes. El foco de la investigación cualitativa (Sampieri, 2013) es comprender y profundizar los fenómenos que se exploran desde la perspectiva de los participantes en un entorno natural y en relación al contexto de investigación.

\subsection{Percurso Metodológico}

De inicio la intervención se aplicará sólo con el sexto año, pues esa es la clase que corresponde al contenido, pero a petición de la profesora regente, la intervención se aplicó en el $6^{\circ}$ y $7^{\circ}$ año, pues la misma había inscrito a dichos alumnos en la OBA - Olimpiada Brasileña de Astronomía, y en el libro didáctico que la escuela esta utilizando, aborda el tema superficialmente en la disciplina de Geografía, con lo que el tema se dividió en cinco clases, abordando el tema desde los primeros de las primeras observaciones del cielo nocturno, y cuestionamientos referentes al formato del planeta, para dar base a los contenidos que serían aplicados en la OBA - Olimpiada Brasileña de Astronomía (Leite \& Colombo Junior, 2020).

La aplicación de la clase en campo fue realizada en el Estadio Municipal Marcio Cassiano da Silva, ubicado en la Avenida Tupiniquins, s / n, en el barrio Nova Jaciara en la ciudad de Jaciara - Mato Grosso.

\section{Resultados e Discussão}

\subsection{Tierra y Universo: Enfoques en la Enseñanza}

Para abordar este tema utilizamos dos clases, y al inicio de cada clase aplicamos un cuestionario abierto para hacer una encuesta de conocimiento previo de los alumnos con tres preguntas para cada clase.

En la primera clase levantamos los siguientes cuestionamientos: ¿Cómo es nuestro planeta, qué formato tiene? Haz un dibujo mostrando cómo crees que es el formato de la tierra y la posición de las estrellas en el cielo. ¿Por qué ocurre el fenómeno del día y de la noche?

Para responder estos cuestionamientos trabajamos con los siguientes temas: Formato de la tierra; Modelos geocéntricos y heliocéntricos; Fenómeno del día y de la noche. Aplicación del contenido en la primera clase presentada para las clases del $6^{\circ}$ y $7^{\circ}$ año. En el libro didáctico no abordaba el contenido con nociones básicas de Astronomía. 
En esta imagen la alumna del $7^{\circ}$ año esta dibujando según su entendimiento, como ella imagina que los habitantes del planeta tierra se quedan en las extremidades Norte y Sur, donde la misma no consideró la fuerza gravitacional (Figura 2a).

Figura 2. (a) Alunma del $7^{\circ}$ año dibujando según su comprensión, como que sería colocada en la parte superior del globo. (b) presentación de la lección para los alumnos de $6^{\circ}$ grado.

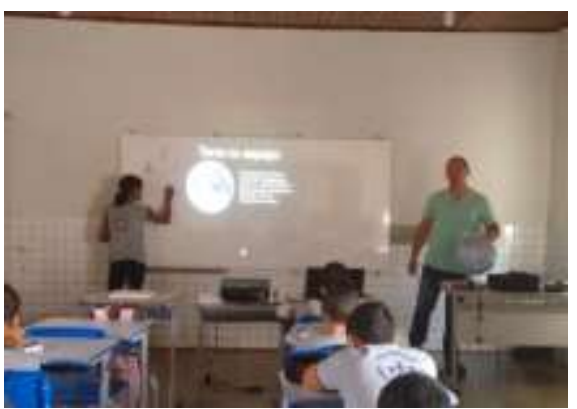

(a)

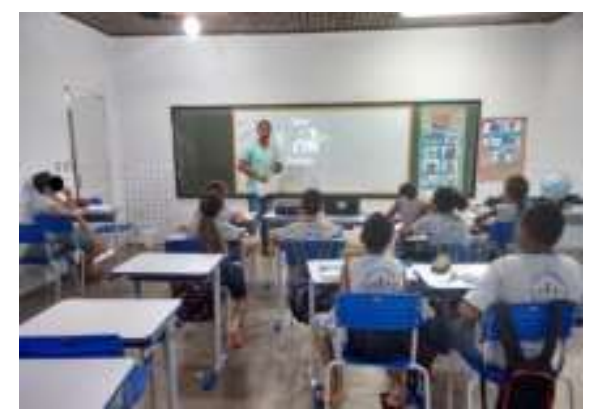

(b)

Fonte: Autores.

Estudiantes de grado $6^{\circ}$ estaban familiarizados con el tema, como que acababa de estudiar acerca de los dos principales movimientos de la tierra en la clase anterior de la disciplina de la geografía (Figura 2b).

En la segunda clase planteamos los siguientes interrogantes: ¿Qué es un eclipse solar? ¿Y qué es un eclipse lunar? ¿Por qué en el mes de Navidad, Brasil esta en la estación verano y Estados Unidos esta en la estación Invierno, siendo que los dos países están del mismo lado geográficamente del planeta? Para responder estos cuestionamientos trabajamos con los siguientes temas: Eclipse solar; Eclipse lunar; y estaciones del año.

Después de la aplicación de los cuestionarios de inicio las clases utilizando presentación en diapositivas, pues en el material didáctico de los alumnos no abordaba el tema. Conocemos algunos mitos de la antigüedad donde creían que eran las respuestas a los cuestionamientos referentes al formato del planeta Tierra, eclipses, por qué ocurría día y noche, vimos también la influencia que la religión tenía sobre la formación intelectual de la sociedad y que en muchas veces obligaban a todos a creer sin cuestionar.

Para la clase que abordaba las estaciones del año, se utilizó una maqueta con bola de isopor, donde fueron utilizadas cuatro bolas de isopor en un soporte de madera en el formato de una "X" en que cada bola fue fijada en cada punta de la "X "En que representaba el planeta tierra en el ángulo de $23^{\circ}$, y una lámpara incandescente en el medio representando el Sol, con eso descubrimos el por qué las estaciones del año son diferentes en el mismo período del año en el polo sur y en el polo norte del planeta tierra.

Con la utilización de una maqueta, fue presentado para los alumnos del $6^{\circ}$, como ocurren los eclipses, después de la clase teórica fue ilustrado para los alumnos que en el eclipse Lunar la Luna queda a la sombra de la del Planeta Tierra, y que en el eclipse Solar, la Luna se queda entre el Planeta Tierra y el Sol proyectando su sobra en el planeta Tierra (Figura 3a). 
Figura 3. (a) Usando un modelo para presentar el eclipse lunar, (b) explicar las estaciones del año, e (c) ilustración de cómo los rayos del sol irradian la tierra y la misma luz del sol llegar a tamaños de diferentes áreas.

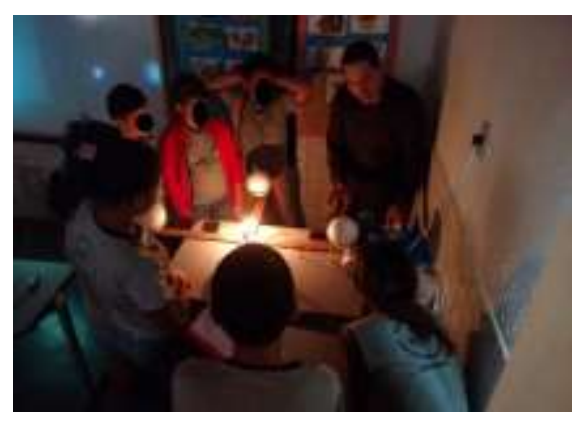

(a)

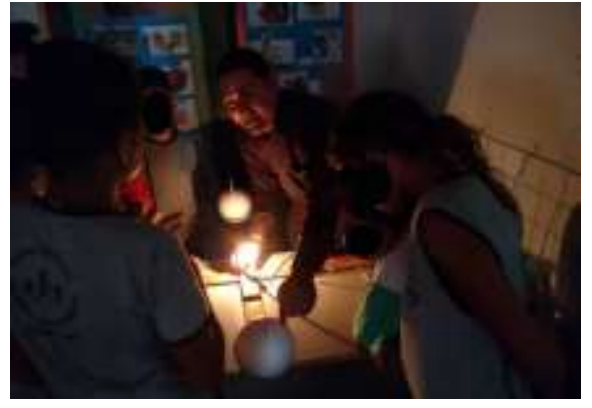

(b)

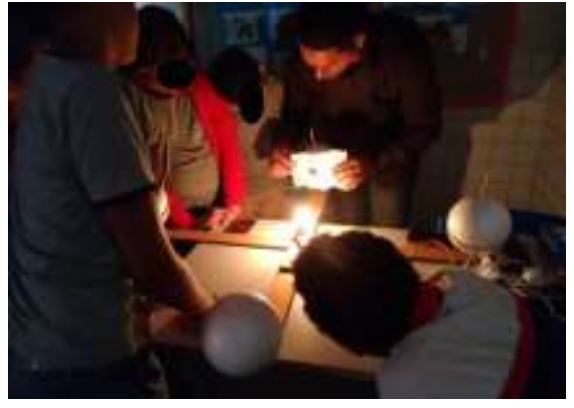

(c)

Fonte: Autores.

La utilización de la maqueta facilitó el discernimiento de los alumnos para visualizar como un observador espacial, la ubicación del planeta tierra en el movimiento de traslación en cada estación del año (Figura 3b)

Ilustración de cómo los rayos solares que alcanzan los trópicos y al mismo tiempo los mismos rayos solares llegan oblicuamente en el Hemisferio Norte y el Hemisferio Sur, y como el Planeta Tierra es inclinado hace que en las extremidades de los hemisferios los rayos solares irradian un área mayor debilitándose en hasta tres veces, haciendo que el clima sea de menor temperatura (Figura 3c).

La clase contó con la participación de los alumnos del $6^{\circ}$ y $7^{\circ}$ año, el contenido propuesto despertó la curiosidad y prendió la atención de los alumnos, daño énfasis para debates y algunas experiencias vividas por los alumnos y también creó expectativa para la próximas clases.

\subsection{Planisfério Celeste}

Para esta clase confeccionamos junto con los alumnos en el aula el Planisferio Celeste, este material didáctico estaba disponible en el sitio de la OBA - Olimpiadas Brasileña de Astronomía, el material es de fácil entendimiento y fácil confección, en él conseguimos identificar las constelaciones del Cielo nocturno, el Planisferio Celeste consiste de un disco de papel donde en su centro está compuesto por un catálogo estelar que se centra en el Polo Celeste Sur donde podemos ver al ojo desnudo las constelaciones en el Cielo nocturno y en su borde marca los doce meses del año y en cada uno el mes marca sus días, y la parte de arriba del disco, colocamos otro disco con las horas de los días, con eso basta elegir el mes, día y horas, posicionar y tendremos las constelaciones que será visible en el Cielo nocturno de aquel día.

Cada alumno confeccionó su propio Planisferio Celeste, para poder observar y aprender los nombres de las constelaciones del Cielo nocturno y debatir en la próxima clase lo que cada uno consiguió identificar la noche anterior (Figura $4 \mathrm{a}, 4 \mathrm{~b}$ e $4 \mathrm{c})$ 
Figura 4: (a) Asistir a los estudiantes en la toma del planisferio Celeste, (b) explicando paso a paso para la confección del Planisferio, e (c) Aluna confeccionando el propio Planisferio Celeste.

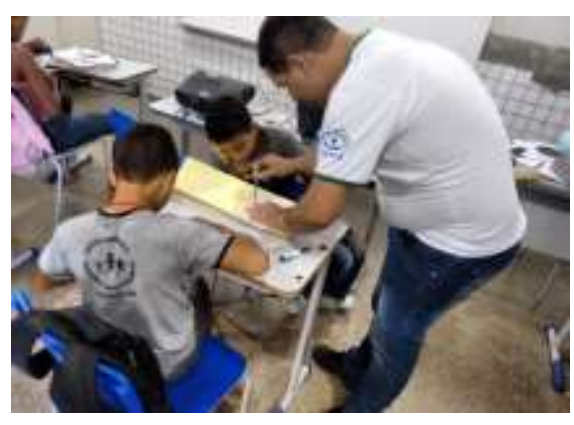

(a)

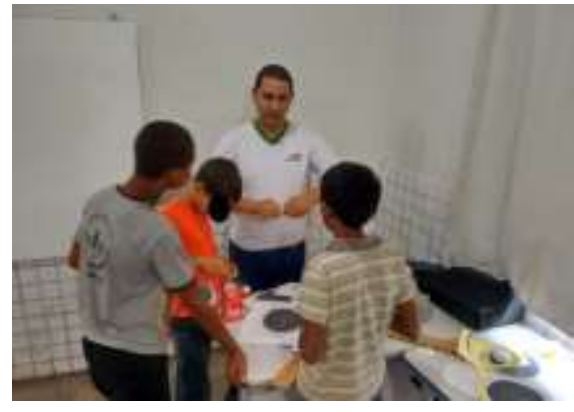

(b)

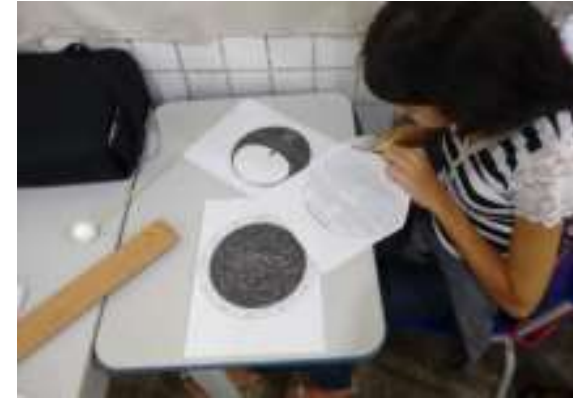

(c)

Fonte: Autores.

Al finalizar la confección del Planisferio Celeste, se presentó para los alumnos de las clases $6^{\circ}$ y $7^{\circ}$ año el software Stellarium ${ }^{\circledR}$, con el propósito de identificar las principales constelaciones del cielo nocturno y ubicar las lanas en el Planisferio Celeste (Figura 5).

Figura 5. Presentación del Software Stellarium®.

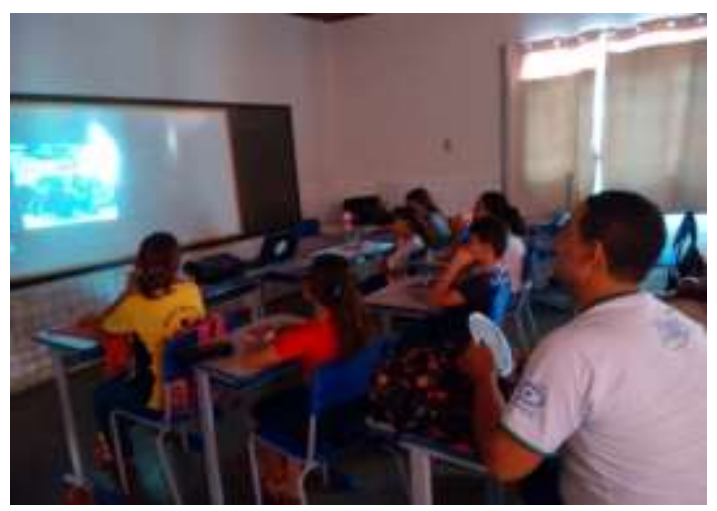

Fonte: Autores.

Con eso los alumnos se fueron familiarizando con el Planisferio Celeste, para que en el período nocturno poder identificar de su casa cuáles las constelaciones visibles aquella noche, que por una feliz coincidencia ocurrir una lluvia de meteoro en las dos próximas noches, pues el Planeta Tierra estaría cruzando por la Tierra la órbita del cometa Halley, y algunos fragmentos en forma de polvo de su caldera caería en el Planeta Tierra y que iba a ser visible en el hemisferio sur aquella noche. Esto trajo más entusiasmo por parte de los alumnos a las clases de astronomía, pena que el cielo nocturno estaba nublado en aquella noche.

\subsection{El Reloj del Sol}

Para la aplicación de esta actividad práctica, volvemos a los primordios de la civilización donde el tiempo era literalmente medido por la luz solar antes de la invención del reloj. Ya habíamos visto que el Sol es la estrella más cercana al planeta Tierra, también vimos sobre los dos principales movimientos de la tierra que es la Rotación y la Traslación, y que a partir del movimiento de rotación del planeta Tierra que ocurre el fenómeno del día y de la noche, y que en algún lugar del 
Planeta Tierra en este momento están recibiendo sus primeros rayos solares del día y que también en algún lugar la noche se está iniciando en ese preciso momento.

La confección del reloj solar fue basada en el ejercicio de clase práctica de la OBA - Olimpiadas Brasileña de Astronomía, que disponibilizó el material para la aplicación en el aula con los alumnos de las escuelas que se inscribieron para las Olimpiadas Brasileña de Astronomía 2015.

El reloj fue confeccionado por los propios alumnos en el aula, y para probar y comprobar la eficacia del material didáctico, los alumnos fueron llevados a la cancha de fútbol sala de la escuela y colocamos los relojes para comparar las horas. El resultado positivo motivó a los alumnos a debates sobre la precisión del reloj solar, y también reforzó a los alumnos que en el globo terrestre existe una línea imaginaria denominada de Línea del Ecuador que divide que divide la región sur y la región norte, pues el reloj solar en la tierra En la región sur del globo terrestre, las horas tienen que comenzar de izquierda a derecha diferente a la región norte del globo terrestre que las horas comienzan de derecha a izquierda, esa regla es algo inherente a la confección del reloj solar, pues la no longitud de esa regla inviabiliza los resultados del reloj solar. Después del término de la confección del reloj solar, los alumnos fueron llevados a la cancha de la escuela para verificar el funcionamiento del mismo (Figura 6).

Figura 6. Comprobando la efectividad del reloj de sol.

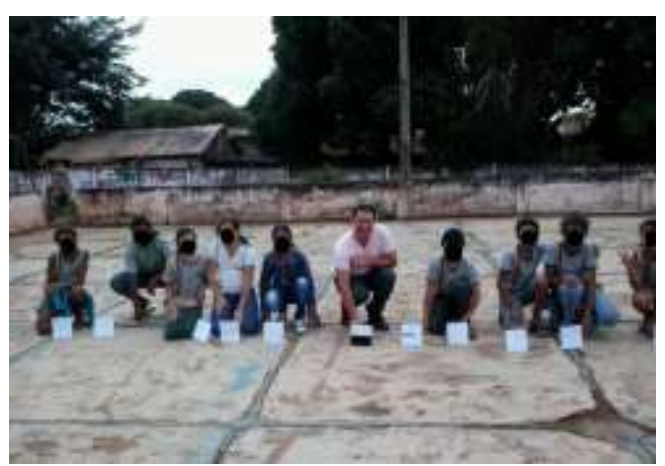

Fonte: Autores.

\subsection{El Sistema Solar}

Esta clase fue el punto culminante de toda intervención, pues siendo el cierre de las clases de toda una temática. El foco del trabajo era presentar para los alumnos los principales cuerpos celestes del Sistema Solar, respetando sus grandezas y distancias entre sí. Pero para la aplicación de este tema ser abordado era necesario un espacio amplio para la exposición de la maqueta, para eso fue montado un planetario en el Estadio Municipal Marcio Cassiano da Silva, en Jaciara - Mato Grosso, donde los alumnos fueron conducidos hasta el referido lugar para estudiar sobre "Las grandezas y distancias de los principales cuerpos celestes del Sistema Solar".

Para poder exponer la maqueta dentro del estadio trabajamos con la siguiente medida, para cada 10 centímetros de esa área, era el equivalente a 1 millón de kilómetros, pero aun usando ese esquema que aparentemente es una distancia considerable, fue necesaria la ampliación en 10 veces los tamaños de los cuerpos celestes, pues si usáramos la misma medida para los planetas, el planeta Mercurio siendo el menor planeta, quedaría con un tamaño un poco menor que un grano de arroz. Pero aún 10 centímetros representando 1 millón de kilómetros, el área disponible en el estadio no fue suficiente para la exposición de todos los planetas en sus respectivas distancias, con lo que las maquetas de los planetas Urano y Neptuno, fueron expuestas fuera de su orden de distancia, pues para eso era necesario que la maqueta del planeta Neptuno estuviera a una distancia de 449 metros lejos de la maqueta del Sol. 
Nuestra expedición por el Planetario allí reproducido inició si por el Sol, y seguía por los planetas, Mercurio, Venus, Tierra, Marte, Júpiter, Saturno, Urano y Neptuno, los alumnos recibieron al inicio de la clase un folder con las principales características de los Planetas y del " Sol, en cada planeta representado la clase era instruida para parar, para la presentación del planeta y en el termino de la explicación un alumno era llamado el "guardián del Planeta", con eso la maqueta de dicho planeta allí representado era entregada al alumno y ese alumno seguía con el resto de la clase para el próximo planeta, esa dinámica fue adoptada para la realización de la comparación de los tamaños de los planetas al final de la expedición.

Al finalizar la expedición, colocamos cada maquete de los planetas frente a frente para la comparación de tamaños entre ellos, después de esa comparación y ver la gran diferencia entre ellos, fue explicado para los alumnos que para la realización de la construcción exacta del planetario basado en los tamaños de las hojas las tablas de los planetas requerir un área mayor, pues para la exactitud de los tamaños y distancias proporcionales, la distancia entre el Sol y el Planeta Neptuno sería cerca de 4,49 kilómetros, y que para atender las distancias allí representadas, las maquetas tendrían que ser 10 veces más pequeña que aquella que cada "Guardián del Planeta" estaba sosteniendo.

Para este modelo, se utilizó para representar el sol una esfera de látex en $139 \mathrm{~cm}$ de diámetro, siendo los planetas proporcional al sol se hacían de arcilla, y representar los anillos de Saturno fue utilizado (Figura 7)

Figura 7. Modelo del sol y los planetas

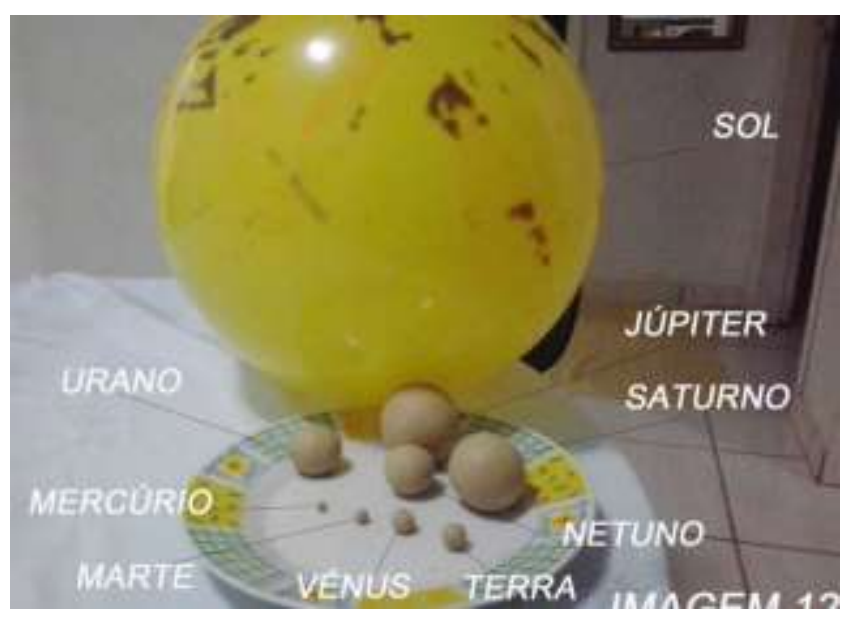

Fonte: Autores.

Este fue el punto de partida para las explicaciones del sol y cada planeta del Sistema Solar, los estudiantes hicieron todo el camino (Figura 8) 
Figura 8. (a) Punto de partida de las explicaciones sobre el Sistema Solar, (b)Tutores de los estudiantes de los planetas mercurio y Venus.

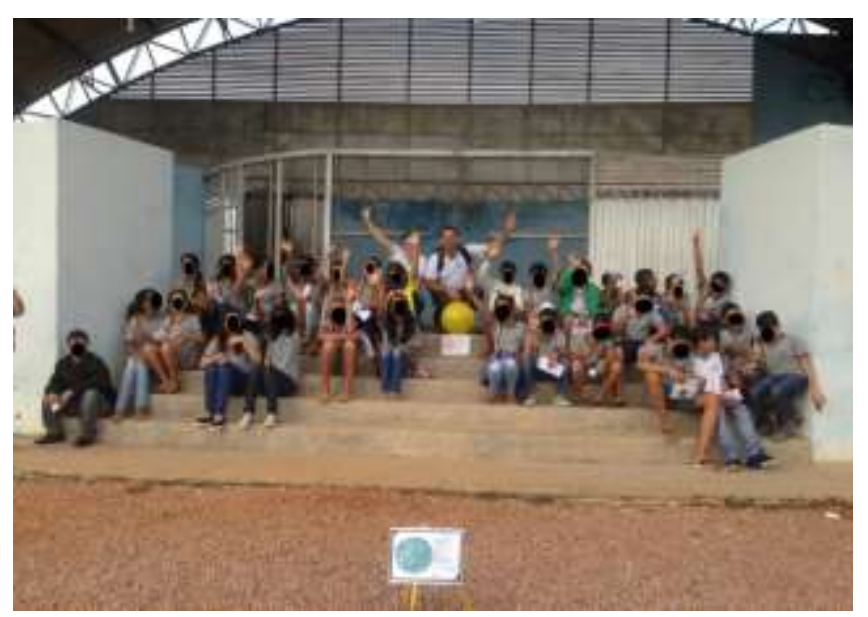

(a)

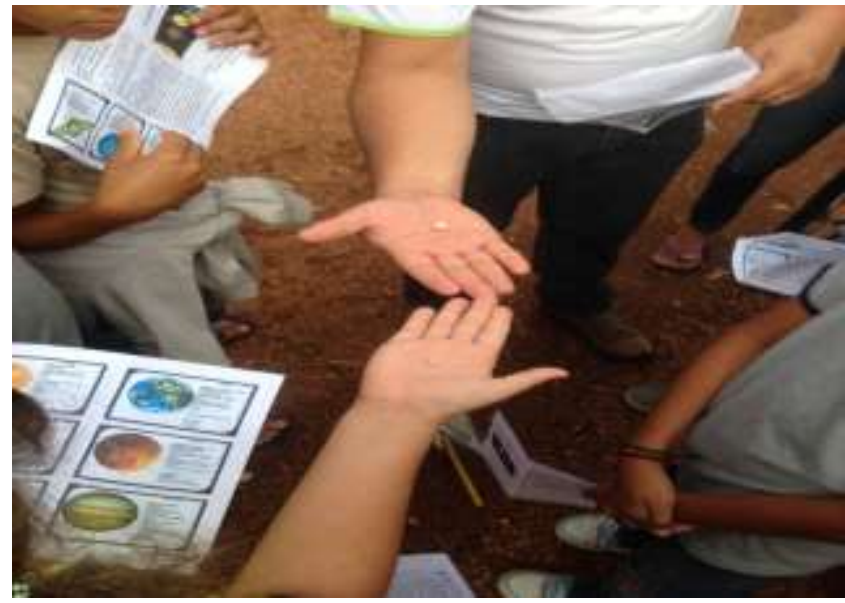

(b)

Fonte: Autores.

En cada parada después de las explicaciones, un alumno se pronuncia en ser el guardián de dicho planeta, aplicamos esa dinámica para obtener mayor participación de los alumnos y para también al final del recorrido hacer la comparación de tamaño de cada planeta (Figura 8b)

En esta figura estamos a la mitad del camino, con la parada en el "gigante gaseoso" el planeta Júpiter, en esta parada miramos hacia atrás y observamos la distancia que estamos del Sol, y cuán pequeño es nuestro Planeta tierra.

Al finalizar la expedición por el planetario, pedimos a los guardianes presentar cada uno su planeta, para hacer la comparación uno del otro, muchos quedaron asombrados por percibir cómo es pequeño nuestro planeta Tierra y quedaron más sorprendidos cuando revelé que aquellas maquetas para atenderlas que distancian propuestas por el planetario allí montado tendría que ser 10 veces menores, disminuyendo aún más el tamaño de nuestro planeta en la inmensidad del espacio (Figura 9a e $9 b)$.

Figura 9. Parada para la explicación del planeta Júpiter, e (b) envío Final en el planetario.

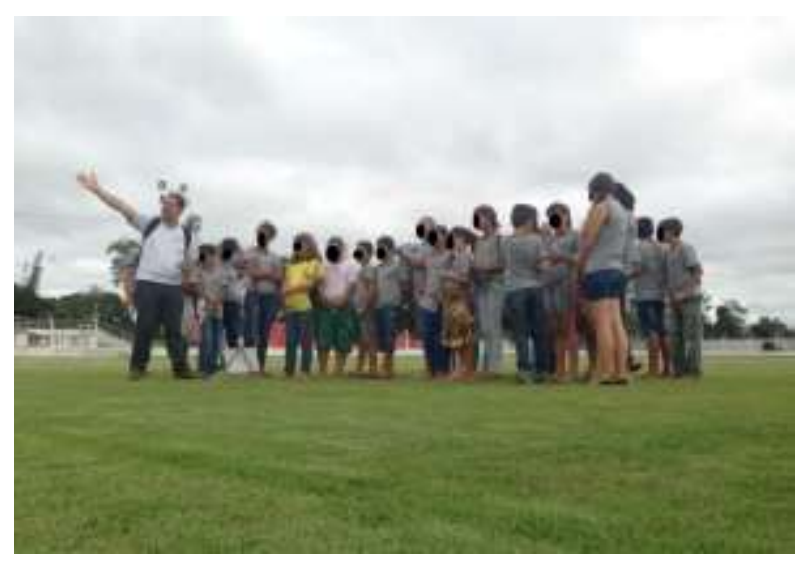

(a)

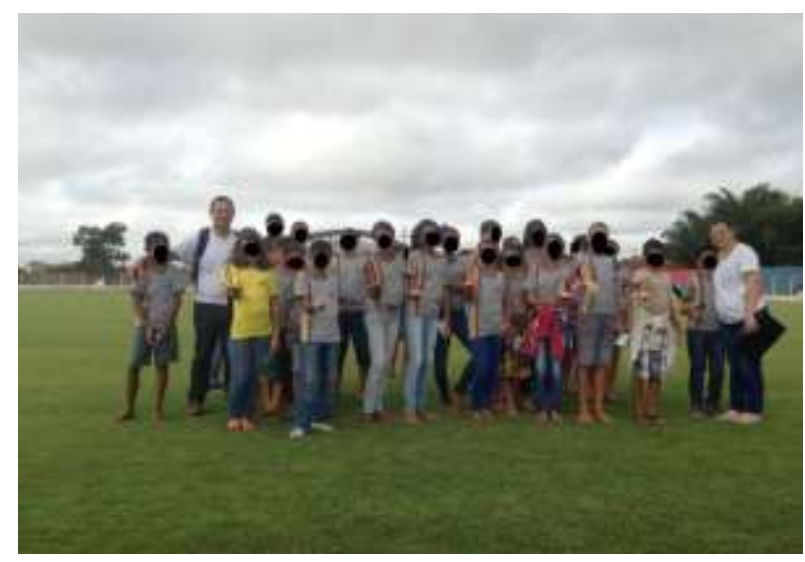

(b)

Fonte: Autores. 
El área utilizada tenía poco más de 170 metros de longitud, y podemos observar que debido a la distancia tuvimos que ampliar la imagen del Sol, teniendo en cuenta siendo un observador que esta en el planeta Neptuno (Figura 10).

Figura 10. Debido a la gran distancia, el sol casi no aparece en la figura.

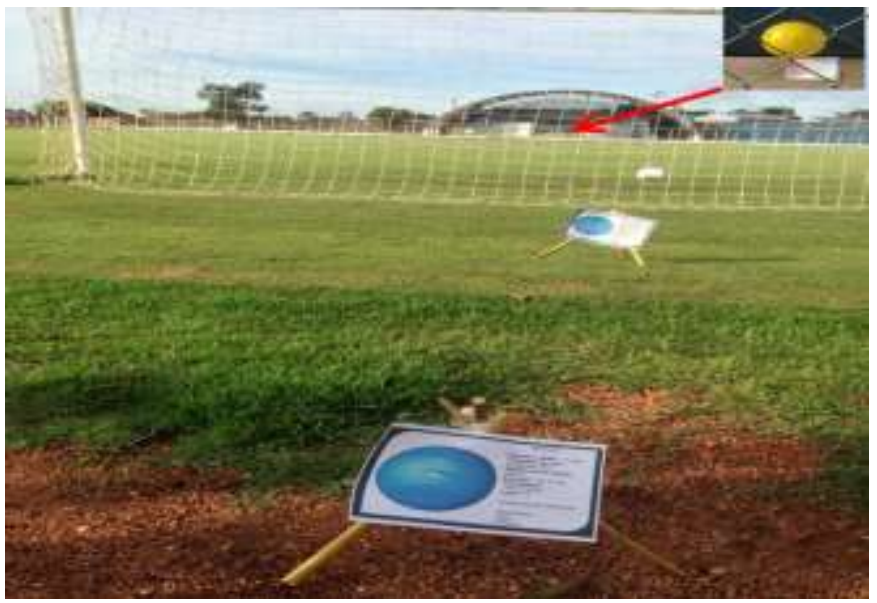

Fonte: Autores.

\subsection{Aplicación de Cuestionario}

Al finalizar todas las clases teóricas y prácticas, fue realizado la aplicación de un cuestionario abierto, se utilizaron las mismas cuestiones aplicadas para el levantamiento del conocimiento previo de los alumnos, con las siguientes cuestiones: ¿Cómo es nuestro planeta, qué formato tiene? Haz un dibujo mostrando cómo crees que es el formato de la tierra y la posición de las estrellas en el cielo. ¿Por qué ocurre el fenómeno del día y de la noche? ¿Qué es un eclipse solar? ¿Qué es un eclipse lunar? ¿Por qué en el mes de Navidad, Brasil esta en la estación verano y Estados Unidos esta en la estación Invierno, siendo que los dos países están del mismo lado geográficamente del planeta?

De inicio los alumnos presentaron resistencia al cuestionario, pues escuela no utilizaba aplicación de cuestionarios como forma de evaluación, y están retornando a esa práctica este año, con lo que se explicó que el cuestionario era algo fuera del currículo de la escuela y que sería para el cierre del contenido de Astronomía (Poffo et al, 2019).

Las clases teóricas tuvieron que ser presentadas con el uso del data show, con presentación de diapositivas pues los libros didácticos utilizados por la escuela abordaban superficialmente el tema sólo en el libro del $6^{\circ}$ año en la disciplina de geografía, por estar acostumbrados sólo con la utilización de pizarra, tiza y libros, los alumnos presentaron una buena disposición para clase, pues sería aplicada de forma atípica para ellos.

De modo participativo y con buen comportamiento, todas las dos clases colaboraron significativamente para el buen curso de la clase. El buen de este tema es que se mecue con la imaginación de los niños, para eso podemos citar algunas grandes películas de ciencia ficción que abordan el tema, con lo que los cuestionamientos fueron apareciendo de modo que iba a construir la enseñanza-aprendizaje de los alumnos.

Un punto que contó con la participación masiva de los alumnos fue el desarrollo de las clases prácticas, en las confecciones de materiales didácticos y presentación de maquetas, el auxilio de los alumnos enriqueció de forma significativa todo el contenido y sumado para el buen andamiento y aplicación de la clase.

El punto alto de toda aplicación de la intervención, la clase en campo fue extremadamente importante y algo que marcó la finalización de la aplicación, los alumnos desplazaron al estadio municipal Marcio Cassiano da Silva situado en la Avenida Tupiniquins s / n, en el barrio Nova Jaciara en la ciudad de Jaciara en el estado de Mato Grosso, donde fue montado 
un planetario con maquetas del Sol y los ocho planetas que componen el Sistema Solar, hasta entonces ellos no tenía idea de las dimensiones y distancias que existen en el espacio.

La elección del estadio fue por el hecho de que sería el único lugar con un área abierta con estructura para el montaje del Planetario, teniendo en vista que los dos últimos planetas no quedaron en las distancias correspondientes al tamaño de la maqueta, pues el espacio aún siendo grande todavía era pequeño para tal aplicación, pero fue presentado de forma didáctica (Tabla 1).

Tabla 1. A continuación está la tabla con las medidas utilizadas para el montaje de cúpula:

\begin{tabular}{|c|c|c|}
\hline ASTRO & CICUNFERENCIA & DISTANCIA \\
\hline Sol & $139 \mathrm{~cm}$ & 0 \\
\hline Mercurio & $0,4 \mathrm{~cm}$ & $5,70 \mathrm{~m}$ \\
\hline Venus & $1,2 \mathrm{~cm}$ & $10,8 \mathrm{~m}$ \\
\hline Tierra & $1,3 \mathrm{~cm}$ & $14,9 \mathrm{~m}$ \\
\hline Marte & $0,7 \mathrm{~cm}$ & $22,7 \mathrm{~m}$ \\
\hline Júpiter & $14,2 \mathrm{~cm}$ & $77,8 \mathrm{~m}$ \\
\hline Saturno & $12,0 \mathrm{~cm}$ & $142,9 \mathrm{~m}$ \\
\hline Urano & $5,1 \mathrm{~cm}$ & $287 \mathrm{~m}$ \\
\hline Neptuno & & $450,4 \mathrm{~m}$ \\
\hline & & \\
\hline
\end{tabular}

Fonte: Autores.

El área disponible dentro del estadio Municipal Marcio Cassiano da Silva fue suficiente para presentar de forma simple y eficaz el trabajo propuesto.

Una de las mayores dificultades encontradas en la aplicación del cuestionario, fue que algunos alumnos son analfabetos funcionales, pues incluso cursando el $6^{\circ}$ y $7^{\circ}$ año, ellos no saben escribir y presentan dificultades en la lectura, y por vergüenza quedan retraídos y no externan la situación. Los alumnos se quedaron con el cuestionario hasta el final, y al entregar, entregó en blanco, con lo que el enfoque tuvo que ser de forma oral, y así fue constado que los alumnos absorbieron el contenido pero tenían dificultades en la escritura (Deosti, et al., 2021).

Al entregar el cuestionario corregido para la profesora regente, la misma resaltó estar muy feliz con el resultado, pues incluso los alumnos no tenían contenido escrito para estudiar para la resolución del cuestionario y no tener el hábito de hacer pruebas, el resultado fue bastante gratificante.

\section{Consideraciones Finales}

La realización de este trabajo buscó tratar con énfasis la disciplina de Astronomía, trayendo de forma sencilla y práctica los conceptos básicos, que al verificar los libros didácticos, poco aborda el tema volviendo cada vez más olvidados en 
la Enseñanza Fundamental, donde una de las barreras más grandes, comienza en la formación del profesor de ciencias, que en su gran parte tiene su formación académica volcada en Ciencias Biológicas.

Este tema surgió el semestre $2^{\circ}$ en la Academia en la forma de un seminario como actividad evaluativa. Para ser un fan de la astronomía y alentado por los maestros a buscar siempre proponiendo las lecciones de práctica del estudiante, buscaba entonces aplicar este contenido más práctico posible, con que los resultados son buenos, porque por ver la participación de los estudiantes en cada actividad se propone en muy gratificante todo lo posible para la preparación de la clase.

Después de haber trabajado con este tema, enriquecido mis conocimientos y creo que he sido capaz de transmitir a los estudiantes y han hecho los resultados esperados. Cada lección dada fue literalmente un viaje por la historia astronómica y la inmensidad del universo, por supuesto hay todavía mucho por hacer y estudio, sabemos que esto es sólo el comienzo de los grandes descubrimientos que están por venir y que sabe sobre el descubrimiento realizado por el descubrimiento estudiantes que participaron en esta conversación.

Nuestra defensa para el trabajo futuro es que los enfoques relacionados con esta temática siempre buscan principalmente estructurar en los estudiantes la asimilación de conceptos básicos de la astronomía, avanzando cuando sea posible a propuestas didácticas que les permitan alfabetizarse científicamente sobre fenómenos relacionados con el sistema solar, movimientos rotacionales. y traslación, fases lunares, influencia de la luna en las mareas, estaciones del año en detrimento de la traslación terrestre, energía producida por las estrellas, evolución del universo desde el Big Bang, etc. También recomendamos que los enfoques relacionados con las macromagnitudes de las estrellas, como el diámetro de los planetas y el sol, y las distancias entre planetas y de la tierra a la luna, se introduzcan en los últimos años de la escuela primaria. Todos estos conceptos son fundamentales para una percepción crítica del universo, y cuando se anclan en la estructura cognitiva de los estudiantes, son capaces de escudarlos de la influencia de los científicos, como está sucediendo hoy en Brasil con la "creencia de la tierra plana".

\section{Referencias}

Ausubel, D. P. (1982). A aprendizagem significativa: a teoria de David Ausubel. São Paulo: Moraes.

Barroso, M. F. B. \& Borgo, I. B. (2010). Jornada no Sistema Solar. Revista Brasileira de Ensino de Física. 32(2), 1-12.

Bogdan, R. \& Biklen, S. (1994). Investigação qualitativa em Educação: uma introdução à teoria e aos métodos. Porto - Portugal: Porto.

Brasil (2017). Base Nacional Comum Curricular: Educação é a Base. Versão final homologada para a Educação Básica.

Chizzotti, A. (2006). Pesquisa qualitativa em ciências humanas e sociais. Petrópolis, RJ: Vozes.

Damasceno Júnior, J. A. \& Romeu, M. C. (2018). O planetário como recurso metodológico para facilitar o ensino de física por meio da ruptura entre o conhecimento científico e o conhecimento comum. Revista Prática Docente (RPD). 3 (1), 231-48.

Deosti, L.; Torres, T. G.; Carvalho, H. A. P. de; Zanatta, S. C.; Silva, A. S. G. da. (2020) Ensinando astronomia para alunos do Ensino Fundamental: uma investigação sobre o Universo. Research, Society and Development. 9 (9), 1-18.

Flick, U. (2013). Introdução à metodologia de pesquisa: um guia para iniciantes. Tradução de Magda Lopes e Dirceu da Silva. Porto Alegre: Penso.

Freire, P. (1996). Pedagogia da Autonomia: Saberes Necessários à Prática Educativa. São Paulo: Paz e Terra.

Gaio, D. C. (2009). Sistema Solar. Cuiabá: UFMT/UAB.

Leite, A. C. \& Colombo Junior, P. D. (2020) Olimpíada Brasileira de Astronomia do Ensino Médio: entre textos e contextos. Research, Society and Development. 9 (9), 1-27.

Moreira, M. A. (2006). A teoria da Aprendizagem Significativa e sua implementação em sala de aula. Brasília: Ed. Universidade de Brasília.

Mourão, R. R. F. (2003). Kepler: A descoberta das leis do movimento planetário. Coleção Imortais da Ciência. Editora Odysseus, Rio de Janeiro.

Mourão, R. R. F. (2000). O Livro de Ouro do Universo. Rio de Janeiro: Ediouro. 
Research, Society and Development, v. 10, n. 10, e275101018854, 2021

(CC BY 4.0) | ISSN 2525-3409 | DOI: http://dx.doi.org/10.33448/rsd-v10i10.18854

Nardi, R. \& Langhi, R. (2009). Ensino da astronomia no Brasil: educação formal, informal, não formal e divulgação cientifica. Revista Brasileira de Ensino de Física. 31 (4). 4402-12.

Nóvoa, A (2009). Professores: Imagens do Futuro Presente. Lisboa; 2009, 27-30.

Pereira, A. S.; Shitsuka, D. M.; Parreira, F. J. \& Shitsuka, R. (2018). Metodologia de Pesquisa Científica. [e-book]. Santa Maria. Ed. UAB/NTE/UFSM. Recuperado de https://repositorio.ufsm.br/bitstream/handle/1/15824/Lic_Computacao_Metodologia-Pesquisa-Cientifica.pdf?sequence=1.

Poffo, R. I. M.; Voelzke, M. R. \& Macêdo, J. A. (2019) Avaliação da aprendizagem de conceitos astronômicos no ensino fundamental. Research, Society and Development. 8 (12), 1-20.

Sampieri, R. H.; Collado, C. F. \& Lucio, M. P. B. (2013). Metodologia de Pesquisa. 5 ed. Porto Alegre, AMGH.

Santana, O. A. \& Figueiredo Neto, A. F. (2009). Ciências Naturais, $6^{\circ}$ ano. 3. ed. São Paulo: Saraiva.

Tignanelli, H. (1998). Didática das Ciências Naturais: Contribuições e Reflexões; in Hilda Weissmann (Org.) Artmed: Porto Alegre. 